\title{
A strategy for electricity buyers in futures markets
}

\author{
Claudio Monteiro ${ }^{1}$, Ignacio J. Ramirez-Rosado ${ }^{2}$, L. Alfredo Fernandez-Jimenez ${ }^{3, *}$ \\ ${ }^{1}$ Department of Electrical Engineering and Computers, University of Porto, 4200-465 Porto, Portugal \\ ${ }^{2}$ Department of Electrical Engineering, University of Zaragoza, 50018 Zaragoza, Spain \\ ${ }^{3}$ Department of Electrical Engineering, University of La Rioja, 26004 Logroño, Spain
}

\begin{abstract}
This paper presents an original trading strategy for electricity buyers in futures markets. The strategy applies a medium-term electricity price forecasting model to predict the monthly average spot price which is used to evaluate the Risk Premium for a physical delivery under a monthly electricity futures contract. The proposed trading strategy aims to provide an advantage relatively to the traditional strategy of electricity buyers (used as benchmark), anticipating the good/wrong decision of buying electricity in the futures market instead in the day-ahead market. The mid-term monthly average spot price forecasting model, which supports the trading strategy, uses only information available from futures and spot markets at the decision moment. Both the new trading strategy and the monthly average spot price forecasting model, proposed in this paper, have been successfully tested with historical data of the Iberian Electricity Market (MIBEL), although they could be applied to other electricity markets.
\end{abstract}

\section{Introduction}

The deregulation process carried out in electricity markets of developed countries in the last thirty years has driven to the establishment of new trading scenarios for buying or selling electricity as any other commodity, although with the special feature of the practical impossibility of storing electric energy for a future consumption. The global electricity market is organized in a sequence of different markets in which the agents trade energy and reserves for different time periods, from mid-term periods covered by forward markets to short and very short-term periods covered by day-ahead, intraday and real-time markets [1].

Electricity forward markets are a set of markets in which electricity purchase/sale contracts are traded years, months, weeks or days prior to the physical delivery of energy. In the medium-term, electricity market agents trade different types of contracts, with delivery periods of different duration (for example, one year, a quarter, a month, etc.). European electricity forward markets trade with several different products [2], the most common are:

- Electricity Forwards: Bilateral contracts adapted to the requirements of agents (electricity buyers or sellers) with physical delivery of electricity at some time in the future at a specified price. They usually are traded in a not organized or standardized market, the so-called "over the counter" (OTC) market. The agents close transactions through intermediaries or brokers (physical or virtual).

- Electricity Futures: A contract under standardised terms and conditions of the exchange, with anonymously and transparency to buyer and seller, and with a specified delivery period and price.
- Electricity Swaps: A swap allows a buyer/seller to set a fixed price for its purchase/sale for an agreed quantity and period of time.

- Electricity Options: An option gives to the buyer/seller the right to purchase/sale a specific quantity of electricity at a predefined price in the future.

On one hand, the participants in forward markets don't need to be able of generating (as producers) or consuming (as retailers or large consumers) electric power because it is possible to trade both physical contracts and financial contracts. On the other hand, in the day-ahead, intraday and real-time markets only electric power producers or consumers can participate. The day-ahead market is usually organized as an auction market in which the price of the electricity for each one of the 24 hours of the next day is established. Electricity prices set in the day-ahead market are called spot prices, and the day-ahead or daily market is also called the spot market.

Forward market prices and spot prices are related to the so-called market Risk Premium (RP). The ex-ante RP has been defined as the difference between forward price and expected spot price [3]; it requires to model the dynamics of the electricity market in order to estimate the future spot price. Since different models could lead to different expected values, it is more common to use the ex-post RP, which is defined as the difference between forward price and realised spot price. The expost RP can be calculated as the sum of the ex-ante RP and the forecast error (difference between the expected or forecasted value and the realised value of the spot price during the delivery period) [4]. 
Electricity forward price reflect the perception of market agents about the spot price in the future, therefore it provides a useful information for the prediction of spot price in the mid-term (from days to a few years) [5, 6]. Thus, futures prices (prices established in electricity futures contracts) have been used as one of the explanatory variables in a short and mid-term forecasting model of the spot prices in a central European electricity market [7].

The Iberian Electricity Market (MIBEL) was created in 2004 as an integration of the Portuguese and Spanish electric power systems and their previous markets. The MIBEL allows any consumer in the Iberian Peninsula (mainland of Portugal and Spain) to purchase electricity from any producer or retailer acting in that region. The daily and intraday markets corresponding to the MIBEL are managed by the company OMIE. Although the MIBEL market includes Spain and Portugal, daily market prices may be slightly different for both countries as a result of the limitation of the power derived from one country to the other in order to don't exceed the capacity of the interconnection power lines. So, electricity prices in MIBEL are identified as SPEL for Spain and as PTEL for Portugal.

The trading of futures contracts in MIBEL is organized through the derivatives exchange market, managed by the company OMIP. The contracts can be base-load or peak-load depending on the hours in the delivery period: all daily hours for base-load contracts or the hours between 8:00 to 20:00 for peak-base contracts. Under an electricity buyer point of view, a physical futures contract in the MIBEL comprises the reception of 1 MW of constant electric power during the corresponding hours in the delivery period (all the hours for base-load contracts or 12 hours per day for peak-base contracts). The delivery period is stabilised according the maturity of the contract. These maturities can be weekly, monthly, quarterly and yearly. Monthly futures contracts in the MIBEL can be traded in the previous six months to the defined as the delivery month.

The relationships between spot, futures and OTC forward prices in the MIBEL has been analysed in [8] revealing a unidirectional short-term causality from the futures price to the proxy of the spot price. Another work that analyses spot and futures prices in the MIBEL for the period 2006-2017 concludes that there some evidence that futures prices can be used to forecast the RP signal [9].

This paper presents an original strategy for buyers in electricity markets. These buyers could be both large consumers and electricity retailers, which can act as agents in the spot and in the futures markets. The strategy is based on the mid-term forecast of the monthly average spot price and the generation of a decision signal for buying electricity in the futures market or wait to buy it in the daily market. The proposed strategy is applied to the MIBEL showing significative improvements related to the conventional strategy of buying electricity only in futures markets.

The structure of the paper is as follows: section 2 presents the mid-term spot price forecasting model and its results in the MIBEL; section 3 describes the proposed strategy for buyers and the results obtained in the MIBEL with the evaluation of the RP; finally, section 4 presents the conclusions.

\section{Medium-term electricity prices forecasting}

In this paper, we consider an approach for predictive conditional trading of MIBEL SPEL base-load monthly physical futures (called as Futures in the rest of the article), where the buyer of Futures will keep the product until the period of electricity delivery (constant power, delivered during a predefined month). In this approach, the objective is to decide, in each negotiation day (period of six months before the delivery month), if the buyer should buy in advance the electricity in the form of Futures for some period ahead (the holding period is 1 to 6 months), or if he/she should wait for the spot market in the day before each delivery day. In the moment of negotiation, it is unknown the price in the spot market; the benchmark decision about buying the physical electricity as Futures is based on the price of Futures in the moment of negotiation and on whether it will be higher or lower than the monthly average spot price in the delivery month. The approach of this paper consists of forecasting the monthly average spot price, using prices information from the day-ahead electricity market and from the Futures market at the moment of negotiation. We estimate a decision signal for buying Futures (in the negotiation day), based on the difference between Future prices and mid-term electricity spot price forecasts, several months before the delivery. The next section presents the forecasting model. Later, the forecasting model results in the MIBEL will be presented.

\subsection{Forecasting model of monthly average spot prices}

The forecasting model predicts the monthly average spot price (arithmetic average of the hourly prices for all the hours in the month settled in the spot market for Spain) of electricity at the moment of delivery, that is, the monthly average spot price predicted in day $n$ for the delivery month. The input variables of this forecasting model are the following ones:

1. Input variable I1: this variable, Delivery Month $(D M)$, is a number between 1 to 12 , representing the month of the year where the commodity (electricity) of the Futures contract will be delivered.

2. Input variable I2: this variable, $\operatorname{Lag} D$, is the number of days between the negotiation day $n$ and the last negotiation day. The last negotiation day is the last trading day before the first delivery day of the delivery month $D M$. The negotiation day $n$ belongs to the negotiation month. For Futures market, between the negotiation month and the delivery month $D M$ there are 6 possible maturities (1 to 6). The maturity represents the lag between the moment of decision and the moment of consequence (delivery). A maturity of value 5 means that the 
delivery period corresponds to the fifth month after the Futures was traded.

3. Input variable I3: this variable is the average of the Futures prices, negotiated in the last 7 days just before the negotiation day, for the delivery month $D M$. This variable represents the last information about Futures settlement.

4. Input variable I4: this variable is the average of MIBEL SPEL base-load quarterly physical futures prices negotiated in the last 90 days just before the negotiation day $n$, for the delivery quarter that includes the delivery month $D M$. The negotiation day could be any working day of the negotiation month.

5. Input variable I5: This variable is the average spot price in the last 7 days before the negotiation day $n$. The negotiation day could be any day in the negotiation month.

6. Input variable I6: This variable is the weekly differential of the weekly average spot price before the negotiation day $n$. This variable represents the trend of the spot price observed in the negotiation day.

The forecasting model is implemented on a multilayer perceptron (MLP) neural network [10] with a single output (the abovementioned monthly average spot price for the delivery month). We use an MLP with a hidden layer of 13 neurons (twice the number of input variables plus one) and with random weights initiation. Since different training processes of an MLP offer slightly different output values, we use, as a final forecasting value, the ensemble averaging [11] of the outputs of ten training processes of the proposed MLP.

\subsection{Results of the forecasting model}

The forecasting model has been successfully tested with historical data of MIBEL. The forecasting model was trained for all kinds of maturities, named LagM (in this work, 6 maturities). The training dataset corresponded to set of values of the input variables in the period January 2015 to August 2018. The target was the MIBEL SPEL monthly average spot price in the delivery month. For the testing period (rolling period, or rolling forecast), the forecast was carried out for each one of the maturities of Futures for every week day in the testing period (September 2018 to August 2019).

The forecasting model provided 6 forecasts for each day in the testing period, one for each holding period (maturity) from 1 to 6 months ahead. Figure 1 plots the ex-post monthly average spot prices (that is, real values), the forecast of the monthly average spot prices, and the Futures prices negotiated in the negotiation day $n$ for a maturity value of 1 month $(\operatorname{Lag} M=1)$.

The well-known normalized Mean Absolute Percentage Error, MAPE, relatively to the ex-post monthly average spot price, was computed for the monthly average spot price forecast (that appears in Table 1, as Spot Forecast) and for the Futures (that appears in Table 1, as Monthly Futures). This table shows the forecasting results achieved with the proposed forecasting model and those achieved using the Futures price as a forecast of the monthly average spot price.

The MAPE results presented in Table 1, show a general better proximity (lower MAPE) for the monthly average spot price forecast than for the Futures settlement. Comparing the MAPE values, we conclude that the values provided by the forecasting model improves the prediction MAPE from $13.0 \%$ to $11.2 \%$. As expected, the error of forecasted price and the error of Futures settlement is lower for shorter LagM (holding period or forecasting horizon). The monthly average spot price forecast improves the MAPE for almost all the 6 types of maturities.

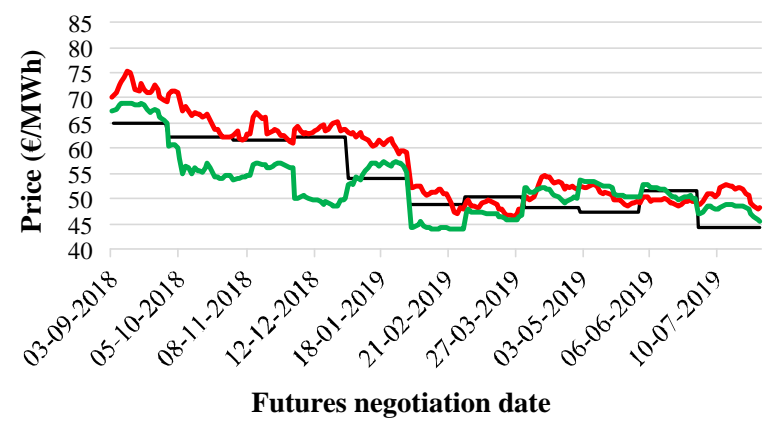

- Ex-post monthly average spot

- Monthly average spot forecast Monthly Futures

Fig. 1. Ex-post monthly average spot, forecast of the monthly average spot, and Futures prices.

Table 1. MAPE values.

\begin{tabular}{|c|c|c|}
\hline LagM & $\begin{array}{c}\text { Spot Forecast } \\
(\mathbf{\%})\end{array}$ & $\begin{array}{c}\text { Monthly } \\
\text { Futures (\%) }\end{array}$ \\
\hline 1 & 7.7 & 6.8 \\
\hline 2 & 7.1 & 9.2 \\
\hline 3 & 8.2 & 12.7 \\
\hline 4 & 12.2 & 14.1 \\
\hline 5 & 15.4 & 15.8 \\
\hline 6 & 15.7 & 17.8 \\
\hline Average & 11.2 & 13.0 \\
\hline
\end{tabular}

\section{Trading strategy of physical futures}

In the context of the trading of physical futures, the general objective is to hedge against the volatility of spot prices. Thus, the objective in the moment of negotiation is to select between two options. First option: to buy electricity as futures, with several days or months in advance. Second option: to accept the price of the spot market settled for each delivery day, that is, wait until the daily market sessions to purchase the electricity.

\subsection{Proposed strategy and evaluation index}

We assume that the forecast of the monthly average spot price is closer to the real value than the Futures price, for the delivery period; thus, we can obtain a signal about whether the Futures prices will be higher or lower than the monthly average spot prices. This signal helps to select between the options above described, that is, buy 
Futures or accept spot (trade in the daily electricity market).

Based on this assumption the trading strategy for buyers consists of buying in the spot market if the forecast of monthly average spot price $\hat{S}_{n, D M}$ is lower than (or equal to) the Futures price $M F_{n, D M}$ in the negotiation day $n$ for the delivery month $D M$. In other case (the forecast of monthly average spot price $\hat{S}_{n, D M}$ is higher than the monthly Future price $M F_{n, D M}$ ), then it consists of buying in the Futures market.

The proposed trading strategy has been evaluated by calculating the ex-post RP. This value evaluates the premium associated with the option of buying Futures instead waiting for and accept spot (in the electricity market). We have used the ex-post RP definition provided in [12], where the RP is evaluated as the difference of the logs of the realized spot price over the delivery period (delivery month) and the monthly futures price for the same period. Thus, a formal description of the Risk Premium $R P_{n, D M}$, in negotiation day $n$, and for the delivery month $D M$, is:

$$
\begin{aligned}
& \text { if } \hat{S}_{n, D M} \leq M F_{n, D M} \text { then } R P_{n, D M}=0 \\
& \text { else } R P_{n, D M}=\ln \left(\bar{S}_{d}^{d \in D M}\right)-\ln \left(M F_{n, D M}\right)
\end{aligned}
$$

where $\bar{S}_{d}^{d \in D M}$ is the average realized spot price computed as the average of the spot price values for all the hours of all the days $d$ belonging to the delivery month $D M$.

In order to evaluate the results of the proposed trading strategy, we compare the RP resulted from the conditional strategy, with the conventional ex-post RP of simply buying Futures in every negotiation day, that corresponds to evaluate always the ex-post RP as $\ln \left(\bar{S}_{d}^{d \in D M}\right)-\ln \left(M F_{n, D M}\right)$. The main difference between the proposed trading strategy and the conventional strategy is that the proposed one consists of a conditional decision, about buying or not, instead the conventional strategy that assumes that buyers always buy Futures. Thus, the benchmark measure of ex-post RP, labelled as RP Futures in charts (in the next section), consists of buying always Futures, for every negotiation day, for all holding periods ( $L a g M$ from 1 to 6).

In order to carry out an overall evaluation of the performance of the proposed trading strategy, the global $\mathrm{RP}$ for buyers is computed as the average of the ex-post RP values for all delivery months and all the maturities, as it is presented in the next section.

\subsection{Results of the proposed trading strategy of physical futures}

The Futures settlement, for the testing period, was generally higher than ex-post monthly average spot price. For this reason, there were losses for buyers (negative ex-post RP). Figure 2 plots the ex-post RP corresponding to the conventional strategy (RP Futures) and the corresponding to the proposed strategy (RP Forecast) for each negotiation day in the testing period with holding period of one month $(\operatorname{Lag} M=1)$.
If the proposed trading strategy, based on predictive information, is applied, the RP is forced to zero (not buying Futures) when the expectation is to lose. The strategy leads to buy Futures if the expectation is to earn. In Figure 2 it is possible to see the evolution of RP along the testing period. The blue dots in Figure 2 represent the RP with the conventional strategy: the RP was negative (losses with the purchase of Futures) until February 2019, but the conditional strategy with forecast (RP Forecast, continuous red line) detected these losses and avoided the buying of Futures. However, in March 2019, buying Futures had positive earnings, but the proposed strategy did not capture this chance to earn. In April and June 2019, the proposed trading strategy expected profits with Futures, winning in June, but losing in April.

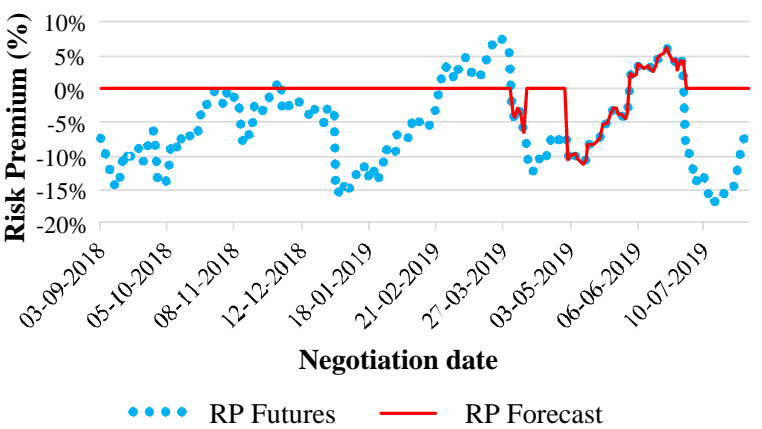

Fig. 2. Ex-post Risk Premium for buyers with a holding period of one month.

In order to evaluate the overall performance of the strategy, it was computed the average ex-post RP for buyers and for each maturity LagM. The average ex-post RP was always negative, and remained negative even with the predictive conditional strategy. However, with the proposed trading strategy, the losses were reduced very notably, from $-11.2 \%$ to $-3 \%$, with significant improvement in all maturities ( $\operatorname{Lag} M)$.

Table 2. Ex-post RP values.

\begin{tabular}{|c|c|c|}
\hline LagM & RP Forecast (\%) & RP Futures (\%) \\
\hline 1 & -0.4 & -5.5 \\
\hline 2 & -1.3 & -9.0 \\
\hline 3 & -2.2 & -13.4 \\
\hline 4 & -5.0 & -14.3 \\
\hline 5 & -6.6 & -15.2 \\
\hline 6 & -6.7 & -16.2 \\
\hline Average & -3.0 & -11.2 \\
\hline
\end{tabular}

In general, we conclude that the proposed trading strategy, using forecasts, have improvements and looseness, depending on several volatility conditions. Therefore, the application of the trading strategy could have an interesting potential as a hedging tactic. In the rolling period used for testing purposes (a representative yearly period with special volatility perturbances in spot and futures prices), the proposed trading strategy provides excellent hedging against losses for buyers. 


\section{Conclusions}

This paper presents a global methodology based on a physical futures trading strategy to help electricity buyers decide whether to buy in the spot market or in the futures market, several months in advance. The trading strategy could be applied for any possible negotiation day, open for monthly futures to be delivered several months ahead (1 to 6 months ahead) and it is based on rules that consider the results of a mid-term forecasting model of the monthly average spot price.

The new trading strategy, proposed in this paper, aims to provide an advantage relatively to the traditional trading buyers (used as benchmark), anticipating the good/wrong decision of buying electricity in the futures market instead in the spot market.

The proposed mid-term monthly average spot price forecasting model is based on the ensemble averaging of 10 MLP neural networks, and uses exclusively available information, at the moment of decision, from the futures and the spot markets.

Both the proposed new trading strategy and the proposed monthly average spot price forecasting model have been successfully tested with historical data from the Iberian Electricity Market (MIBEL), although they could be applied to other electricity markets.

Despite the simplicity of the mid-term forecasting model, its results are promising, proving that it is possible to predict monthly average spot prices with lower MAPE values than the achieved using Futures prices as the forecast of the spot prices. The proposed forecasting model achieves better results (it improves the MAPE values) almost in all the studied maturities.

The ex-post RP achieved with the proposed trading strategy is compared with the achieved with a conventional trading strategy (the buyer always buys in the futures market). The results of this comparison show that the proposed trading strategy achieves an overall benefit compared with the conventional strategy: the negative RP (buyer losses) notably decreases in absolute value, that is, the proposed strategy achieves a significant reduction in buyer losses compared to the losses of those who follow the conventional strategy.

Acknowledgments. The authors would like to thank the "Ministerio de Economia, Industria y Competitividad" of the Spanish Government for supporting this research under the project ENE2016-78509-C3-3-P and the ERDF funds of the European Union.

\section{References}

1. D.W. Edwards. Energy Trading \& Investing (McGraw-Hill, New York, 2009).

2. Economic Consulting Associates (ECA). European Electricity Forward Markets and Hedging Products-State of Play and Elements for Monitoring. Technical Report (ACER, London, 2015)

3. F.E. Benth, A. Cartea, R. Kiesel, J. Bank Financ. 32, 2006-2021 (2008)

4. A. Cartea, P. Villaplana, Quantitative Energy Finance, 215-236 (Springer, New York, 2014)

5. H. Bessembinder, M.L. Lemmon, J. Financ. 57, 1347-1382 (2002)

6. R. Huisman, M. Kilic, Energy Econ. 34, 892-898 (2012)

7. R. Steinert, F. Ziel, Energy J. 40, 105-127 (2019)

8. J.M. Ballester, F. Climent, D. Furio, Rev. Esp. Financ. Contab. 45, 135-153 (2016)

9. M. Ferreira, H. Sebastião, J. Energy Markets 11, 61-82 (2018)

10. S. Haykin, R. Lippmann. Neural Networks: A Comprehensive Foundation (Prentice Hall, Upper Saddle River, 1999)

11. S. Hashem, Neural Netw. 10, 599 (1997)

12. R. Weron, M. Zator, Energy Econ. 44, 178-190 (2014) 